\title{
Effect of a Malaria Control Program on the Prevalence of Malaria, Fever and Anaemia in Children under Five Years in the Hohoe Municipality of Ghana: A Comparative Analysis of Cross-Sectional Surveys
}

\author{
Margaret Kweku1 ${ }^{*}$, Eric Kwaku Appiah², Wisdom Takramah1, Yeetey Enuameh1, \\ Ishmael Norman ${ }^{1,3}$, Fred Binka ${ }^{4}$ \\ ${ }^{1}$ School of Public Health, University of Health and Allied Sciences, Ho, Ghana \\ ${ }^{2}$ Department of Epidemiology, Surveillance and Disease Control, 37 Military Hospital, Accra, Ghana \\ ${ }^{3}$ Institute for Security, Disaster and Emergency Studies, Sandpiper Place, Langma, Ghana \\ ${ }^{4}$ Chancellery, University of Health and Allied Sciences, Ho, Ghana \\ Email: *margaretkweku069@gmail.com
}

Received 2 November 2015; accepted 19 December 2015; published 22 December 2015

Copyright (C) 2015 by authors and Scientific Research Publishing Inc.

This work is licensed under the Creative Commons Attribution International License (CC BY). http://creativecommons.org/licenses/by/4.0/

(c) (i) Open Access

\section{Abstract}

Background: Malaria and anaemia continue to adversely impact the health of children in Ghana. Hohoe is an area of intense and prolonged, seasonal malaria transmission. In 2006, malaria control programme activities which provided In-secticide Treated Bed-Nets (ITNs) to resident children under five years and Artemisinin Combination Therapies (ACTs) for the management of malaria were introduced into the Hohoe Municipality. Before the introduction of the control programme, baseline surveys were carried out in communities in the Hohoe municipality to determine the prevalence of malaria, fever, anaemia, malaria parasite density, gametocytaemia and ITN ownership and use in June and November 2006 ahead of the intervention programme. Similar surveys were conducted in $\mathbf{2 0 1 0}$ after the intervention to assess changes in the earlier indicators in the same communities. This report presents an evaluation of the intervention by comparing findings before and after the malaria control interventions. Methods: In 2010, two communitybased surveys were carried out in thirty communities among children aged five years and below. The first one was at the beginning of the rainy and high malaria transmission season in June and the other was in November at the end of the rainy season. The surveys were to determine the prevalence of malaria, fever, anaemia and ITN ownership, use and effectiveness among children less

"Corresponding author. 
than 5 years. Data were collected in the form of interviews using questionnaire and collection of biological samples. Findings were compared to those similar surveys conducted in the same communities and age groups in 2006. Pr-testi was used to analyze two sample tests for proportions and t-test was used for means. Findings: Malaria prevalence decreased by $20 \%$ [9.0\% vs. 7.2\%; $p=$ 0.0.40], fever decreased by $47.8 \%[2.3 \%$ vs. $1.2 \% ; p=0.008]$ and anaemia decreased by $32.9 \%$ [7.8\% vs. 5.3\%; $p=0.002]$. ITN ownership increased by $67.9 \%$ [20.8\% vs. $64.8 \%$; $p<0.001]$, ITN use increased by $64.2 \%$ [15.1\% vs. $42.2 \% ; p<0.001]$ and ITN effectiveness increased by $41.7 \%$ [20.0\% vs. 34.3\%; $\mathbf{p}<\mathbf{0 . 0 0 1 ]}$. The proportion of children with high density parasitaemia reduced by $17.9 \%$ [2.8\% vs. $2.3 \% ; p=0.223]$. Gameto-cytaemia decreased by $82.5 \%$ [0.8\% vs. $0.14 \% ; p=$ 0.002. Similarly data comparing 2006 and 2010 post-rainy season showed that malaria prevalence decreased by $16.8 \%$ [ $40.4 \%$ vs. $33.6 \%$; $p<0.001$ ], fever decreased by $14 \%$ [5.0\% vs. $4.3 \%$; $p=$ $0.347]$ and anaemia decreased by $64.2 \%[12 \%$ vs. $4.3 \%$; $<<0.001]$. The proportion of children with high density parasitaemia also reduced by $69.5 \%$ [10.5\% vs. $3.2 \% ; p<0.001]$ and gametocytaemia decreased by $78.5 \%[0.8 \%$ vs. $0.17 \% ; p=0.008]$. Conclusion: This study suggested that there was an association between the current intervention and reduction in the prevalence of malaria, fever and anaemia. High parasite density and gametocytaemia prevalence have also significantly reduced over the five years of the introduction of the control measures. There has also been a significant increase in the ownership and use of ITNs.

\section{Keywords}

Malaria, Parasitaemia, Mean Parasite Density, Anaemia, Fever, Malaria Transmission, Gametocytaemia, Insecticide Treated BedNets, Children under Five Years, Hohoe Municipality, Ghana

\section{Introduction}

Globally malaria defined as a fever with any parasitaemia and anaemia defined as haemoglobin concentration $(<8.0$ $\mathrm{g} / \mathrm{dl}$ ) are major causes of morbidity and mortality among children. Anaemia is one of the complications of malaria [1]-[3]. Malaria kills more than one million children (2800 per day) each year in Africa alone [4]. SubSaharan Africa is the region with the highest malaria infection rate, killing at least one million people each year [5]. Malaria is a focal problem in Ghana with an estimated 7.2 million cases in 2006, accounting for 3\% of the total for the World Health Organization (WHO) African region [6]. There is no evidence of reduction in malaria cases between 2001 and 2007 and reported deaths increased in 2007 [5]. The Ministry of Health (MOH), Ghana, estimates that 3 - 3.5 million cases of suspected malaria are reported each year in public health facilities, representing $30 \%-40 \%$ of out-patient attendance [3]. Of this figure, over 900,000 are children below five years of age. The Hohoe Municipality has an intense and prolonged seasonal malaria transmission. The prevalence of malaria and anemia in children 5 years and below was found to be high [7]. Malaria is the leading cause of outpatient department visits (46.21\%) and accounts for 30\% of the total admissions. Malaria accounts for $16 \%$ and anaemia $12 \%$ of the top ten causes of death [8]. Plasmodium falciparum is the dominant malaria parasite in the area [9].

The 2000 to 2010 strategic plan gave direction to the Ghana Malaria Control [10]. To achieve the targets set by the plan, Ghana implemented a malaria control strategy involving multi- and inter-sectoral partnerships working to an agreed plan. The plan was to reduce illness and death due to malaria by $50 \%$ by the year 2010 [11]. Though Ghana has made progress implementing its National Malaria Control Programme (NMCP), gaps still existed in achieving set targets. The May 2006 Abuja declaration entreated countries to put in place new measures to reduce malaria burden by $75 \%$ by the year 2015 [11] [12]. These strategies implemented to achieve these goals included, preventive measures through the use of insecticide treated bed-nets (ITNs), long lasting insecticide nets (LLINs) and intermittent preventive treatment (IPT) in pregnancy (IPTp), in infants (IPTi) and in children (IPTc) and indoor residual spraying (IRS). Other curative measures were the early detection and appropriate prompt treatment with Arthemisinin-based combination therapy (ACT) [13]. The intervention adopted depended on outcomes of situational analysis and assessments, environmental, social and economic factors, as 
well as vector characteristics of each locality [14].

In 2006, as part of the NMCP strategy to reduce the prevalence and incidence of malaria and anaemia, ACTs became the first line drugs for treating uncomplicated malaria in Ghana. That same year, ITNs were distributed at no cost to households in four administrative regions of Ghana as part of the National Immunization Days (NIDs) campaign in December. The Volta Region was the first to benefit from these interventions. The NMCP with support from the Global Fund has since 2006 regularly supplied ITNs at reduced costs to households with children aged five years and below countrywide [15]-[17].

Five years after the introduction of these malaria control interventions, it is imperative to find out their impact on malaria and anaemia prevalence in the municipality. The study therefore compared the prevalence of malaria, fever and anaemia determined in children aged five years and below in 2006 and 2010 in cross-sectional surveys in communities within the Hohoe municipality.

\section{Materials and Methods}

\subsection{Study Area}

The study area is Hohoe municipality which is one of the twenty-five administrative districts of the Volta Region. The municipality is located in the Volta region of Ghana with a population of 167,000 people. Hohoe the municipal capital has a population of 63,000 people [18]. Just like most of Ghana, the municipality has two main seasons, the wet and dry. The major wet season is from April to July and the minor one from September to November. The rest of the year is relatively dry. The climate is tropical with temperatures varying between $22^{\circ} \mathrm{C}$ and $37^{\circ} \mathrm{C}$. The average annual rainfall in the municipality is $1592 \mathrm{~mm}$ with approximately $1296 \mathrm{~mm}$ of rain falling between April and October [17]. Malaria is hyper endemic in the study area but with seasonal peaks. The entomological inoculation rate (EIR) for the study area is approximately 65 (95\% CI: 0 - 143) infectious bites per person per year [17]. Malaria transmission occurs throughout the year with seasonal peaks, coinciding with the period of the rains (high malaria transmission begins from June and ends in November).

\subsection{Study Design}

The study design was cross-sectional. Data were collected in the form of interviews and biological samples. The study population was all children in the selected communities aged 6 to 59 months who were eligible and their parents/guardians consented to participate.

Cross sectional surveys of children under five years were carried out in June 2010 the beginning of the high transmission season (pre-rainy season) and at the end of the high transmission season in November 2010 (postrainy season). It involved asking parents/guardians of the children questions and collection of finger prick blood from the children for analysis. During these surveys, temperature and weight were measured and a finger prick blood sample was collected for determination of malaria parasitaemia and haemoglobin ( $\mathrm{Hb}$ ) concentration levels. Information was obtained on ITN ownership, use and effectiveness (defined as ITNs being able to kill or prevent mosquitoes from biting). The data obtained was compared with that obtained in June and November 2006.

\subsection{Laboratory Methods}

Malaria was measured by examination of thick blood films for malaria parasites. A sample was considered negative only after 200 high power fields had been read. Parasite counts were converted to parasites per micro liter $(\mu \mathrm{l})$, assuming a white blood cell count of 8000 leukocytes per $\mu \mathrm{l}$ of blood. If there was a discrepancy in the findings in a slide between the two initial technicians (positive or negative or a $50 \%$ or more difference in parasite density) a third, more senior microscopist read the slide and his reading was deemed to be the correct reading. A senior microscopist from the Noguchi Memorial Institute of Medical Research (NMIMR, Ghana) examined all the positive blood films and a $20 \%$ random sample of negative blood slides for quality control. Fever was measured using electronic thermometer and haemoglobin was measured using Hemocue ${ }^{\circledR}$ Photometer (Leo Diagnostics, Sweden).

\subsection{Sampling and Sample Size Calculation}

Thirty communities were selected by one-stage cluster sampling during both 2006 and 2010 surveys from a 
sample frame of all communities in the municipality from the 2000 census. This sampling approach was used to ensure a fair representation of communities in the Municipality in the study. The sample size was estimated on the basis of the following: using a 95\% confidence level $(\mathrm{Z})$ and power of $80 \%$, the prevalence $(\mathrm{P})$ of malaria in children aged less than 5 years in June 2006 (end of dry season) was $9.0 \%$. The worst acceptable prevalence of malaria to be 5.0\% using Epi-Info STATCALC software, sample size was calculated for the cross sectional studies as 1374 children aged less than 5 years.

\subsection{Data Management}

Data from participants were recorded on specified forms and were checked by field supervisors and data manager for consistency and accuracy. All data were entered twice into a database using EPI Data software. The accuracy of data input was checked and validated using customized validation programmes. The cleaned data were converted to Stata version 10 file by a statistician (Stata Corporation, Texas, USA) prior to analysis.

\subsection{Statistical Analyses}

The primary trial endpoints were the prevalence of malaria, fever (defined as axillary temperature $\geq 37.5^{\circ} \mathrm{C}$ ) and anaemia, defined as an $\mathrm{Hb} \leq 8.0 \mathrm{~g} / \mathrm{dl}$. Proportion of children with malaria parasitaemia, fever and anaemia in 2010 were compared with that of the pre- and post-rainy season periods of 2006. Analysis of the primary and secondary outcomes was carried out on an intention-to-treat basis in which all children screened were included. Proportions of outcomes were analyzed using $\mathrm{z}$ test for two sample test of proportion. Differences in means were calculated using $t$ test for comparison of two means. All analyses were done with STATA software version 10.0 .

\subsection{Ethical Issues}

The study was approved by the Ethical Review Committee (ERC) of the Ghana Health Service, Ministry of Health (GHS/MOH). The identification number for the clearance was GHS-ERC: 32/4/10. Before the commencement of the study, permission was sought from the Municipal Health Management Team (MHMT) and the municipal administration. Permission was also sought from the chiefs and elders in the selected communities. The objectives and the benefits of the study were explained to them. A written informed concern was obtained from the parents/guardians of the children. All the information collected was treated confidentially and used for research purposes only.

\section{Results}

The study population was two thousand, one hundred and fifty-five (2155) and one thousand seven hundred and seventy-eight (1778) children in June and November 2010 respectively. The population for June and November 2006 were 1717 and 1435 children respectively. Overall, sex, mean age and weight distribution were similar in both groups. ITN ownership, use and effectiveness was significantly higher in the 2010 survey compared to 2006 [64.8\% vs. 20.8\%; p < 0.001], [42.2\% vs. 15.1\%; p < 0.001] and [34.3\% vs. 20.0\%; p < 0.001] respectively (Table 1).

\subsection{Comparison of 2006 and 2010 Pre-Rainy Season Parasitaemia, Fever and Anaemia}

The overall parasitaemia prevalence in the study sample during the pre-rainy season survey in 2006 and 2010 decreased by $20 \%$. The difference was statistically significant $[9.0 \%$ vs. $7.2 \%$; $=0.040]$. Fever significantly decreased by $47.8 \%$ [ $2.3 \%$ vs. $1.2 \%$; $\mathrm{p}=0.008$ ). Anaemia prevalence significantly decreased by $32.9 \%[7.9 \%$ vs. 5.3\%; p = 0.001] respectively. Mean Hb significantly improved from $9.27 \mathrm{~g} / \mathrm{dl}$ in 2006 to $9.7 \mathrm{~g} / \mathrm{dl}$ in 2010 (p < 0.001). Mean parasite density at the pre-rainy season survey was 14,348 $(25,313)$ in 2006 and $28(7378)$ in 2010. Though there was a reduction in proportion of children with high density parasitaemia from $2.8 \%$ in 2006 to $2.3 \%$ in 2010, the difference was however not statistically significant $(\mathrm{p}=0.324)$ (Table 2). Gametocyte prevalence decreased by $82.5 \%$ [ $0.8 \%$ vs. $0.14 \%$; $p=0.002]$. ITN ownership increased by $67.9 \%$ [ $20.8 \%$ vs. $64.8 \%$; $\mathrm{p}<$ $0.001]$, ITN use increased by $64.2 \%$ [ $15.1 \%$ vs. $42.2 \%$; $<0.001]$ and effectiveness was increased by $41.7 \%$ [20.0 vs. 34.3; $\mathrm{p}<0.001$ (Table 1 ). 
Table 1. Characteristics of children in the two studies seasons.

\begin{tabular}{cccc}
\hline & $\begin{array}{c}\text { Pre-rainy season } 2006 \\
\text { No (\%) }\end{array}$ & $\begin{array}{c}\text { Pre-rainy season 2010 } \\
\text { No (\%) }\end{array}$ & p-value \\
\hline Number screened & $1717(100)$ & $2155(100)$ & 0.312 \\
Mean age (in months) (SD) & $27.3(15.4)$ & $27.8(15.5)$ & 1.000 \\
Age groups (months) & & & 0.216 \\
$\mathbf{6}$ - 11 & $321(18.0)$ & $389(18.0)$ & 0.362 \\
$\mathbf{1 3}$ - 23 & $454(25.4)$ & $510(23.7)$ & 0.056 \\
$\mathbf{2 4}$ - 35 & $399(22.3)$ & $454(21.1)$ & 0.003 \\
$\mathbf{3 6}$ - 47 & $319(17.8)$ & $436(20.2)$ & $366(17.0)$ \\
$\mathbf{4 8}$ - 59 & $243(13.6)$ & $11.3(3.3)$ & 0.381 \\
Mean weight (Kg) (SD) & $12.8(2.7)$ & $1031(47.8)$ & 0.001 \\
Sex (male) & $880(49.2)$ & $1396(64.8)$ & 0.001 \\
Own ITN & $357(20.8)$ & $910(42.2)$ & 0.001 \\
Use ITN & $260(15.1)$ & $740(34.3)$ & \\
Effectiveness & $344(20.0)$ & &
\end{tabular}

Table 2. Comparison of the primary and key secondary outcomes of the two studies at the beginning of the rainy seasons.

\begin{tabular}{ccccc}
\hline & Pre-rainy season 2006 & Pre-rainy season 2010 & z & p-value \\
\hline Number screened & 1717 & 2155 & & 0.008 \\
Fever & $42(2.3 \%)$ & $25(1.2)$ & 2.64 & 0.040 \\
Any malaria parasitaemia & $156(9.0 \%)$ & $156(7.2)$ & 2.05 & 0.324 \\
High density malaria parasitaemia & $50(2.8 \%)$ & $49(2.3)$ & 0.99 & 0.002 \\
Gametocytaemia & $14(0.8 \%)$ & $3(0.14)$ & 3.11 & 0.002 \\
Anaemia (Hb < 8.0 g/d) & $135(7.8 \%)$ & $114(5.3)$ & 3.16 & 0.001 \\
Mean parasite density (SD) & $14,348(25,313)$ & $28(7378)$ & & 0.001 \\
Mean Hb (SD) & $9.27(1.29)$ & $9.7(1.3)$ & & \\
\hline
\end{tabular}

\subsection{Parasitaemia, Fever and Anaemia Prevalence during the Post-Rainy Season Survey}

The overall parasitaemia prevalence in the study sample during the post-rainy season survey in 2006 and 2010 significantly decreased by $16.8 \%$ [ $40.4 \%$ vs. $33.6 \%$; $p<0.001$ ]. Fever prevalence decreased by $14 \%$ [5.0\% vs. $4.3 \%, \mathrm{p}=0.347$ ] however, the difference is not statistically significant. Anaemia prevalence significantly decreased by $64.2 \%$ [12\% vs. 4.3\%; p < 0.001]. Mean Hb significantly improved from $9.36 \mathrm{~g} / \mathrm{dl}$ in 2006 to $9.6 \mathrm{~g} / \mathrm{dl}$ in $2010(\mathrm{p}=0.001)$. Mean parasite density at post-rainy season significantly decreased from $9516(25,115)$ in 2006 to $1578(10,334)$ in 2010 ( $<<0.001)$. The proportion of children with high density parasitaemia significantly reduced by $69.5 \%$ [10.5\% vs. 3.2\%; p < 0.001]. Gametocytaemia also significantly decreased by $78.5 \%$ [0.8\% vs. $0.17 \%$; $\mathrm{p}=0.008]$ (Table 3 ).

Figure 1 shows that ownership of ITNs was about $65 \%$, use was $42 \%$ and effectiveness was $34.3 \%$. ITN ownership inched from $20.8 \%$ in 2006 to $64.8 \%$ in 2010 and ITN use increased from $15.1 \%$ in 2006 to $42.2 \%$ in 2010 and effectiveness of ITNs rose from 20.0 in 2006 to 34.3\% in 2010.

Figure 2 reveals that the prevalence of malaria has reduced from $9.0 \%$ (2006) to $7.2 \%$ (2010) at the beginning of the rainy season and about $17 \%$ reduction at the end of the rainy season (from $40.4 \%$ to $33.6 \%$ ). 
Table 3. Comparison of the primary and key secondary outcomes of the two studies at the end of the rainy seasons.

\begin{tabular}{ccccc}
\hline & Post-rainy season 2006 & Post-rainy season 2010 & $\mathrm{z}$ & $\mathrm{p}$-value \\
\hline Number screened & $1435(100)$ & $1778(100)$ & & 0.347 \\
Fever (Temp $\left.\geq 37.5^{\circ} \mathrm{C}\right)$ & $71(5.0)$ & $77(4.3)$ & 0.94 & 0.001 \\
Any malaria Parasitaemia & $580(40.4)$ & $590(33.6)$ & 3.98 & 0.001 \\
High density malaria Parasitaemia & $151(10.5)$ & $56(3.2)$ & 8.37 & 0.008 \\
Gametocytaemia & $12(0.8)$ & $3(0.17)$ & 2.65 & 0.001 \\
Anaemia (Hb $<$ 8.0 g/dl) & $172(12.0)$ & $76(4.3)$ & 8.12 & 0.001 \\
Mean parasite density (SD) & $9516(25,115)$ & $1578(10,334)$ & & 0.001 \\
Mean Hb (SD) & $9.36(2.63)$ & $9.6(1.3)$ & & \\
\hline
\end{tabular}

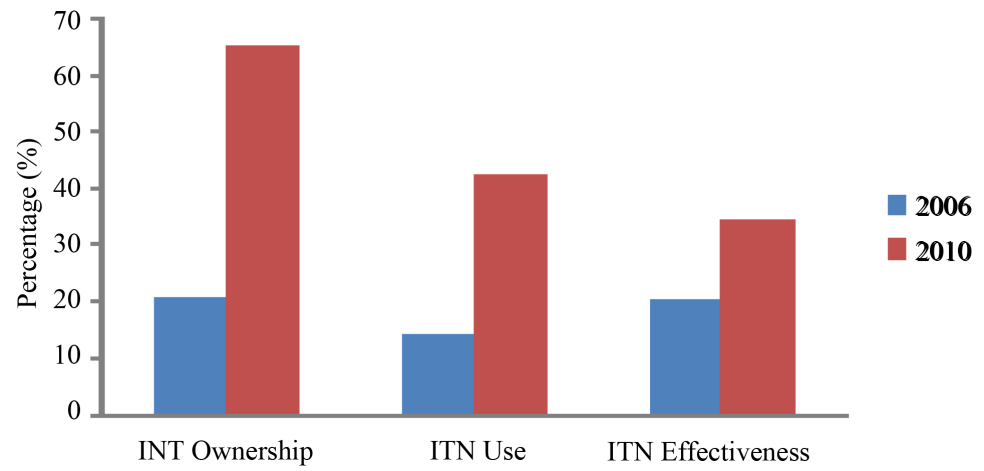

Figure 1. Comparison of ITN ownership, use and effectiveness between 2006 and 2010.

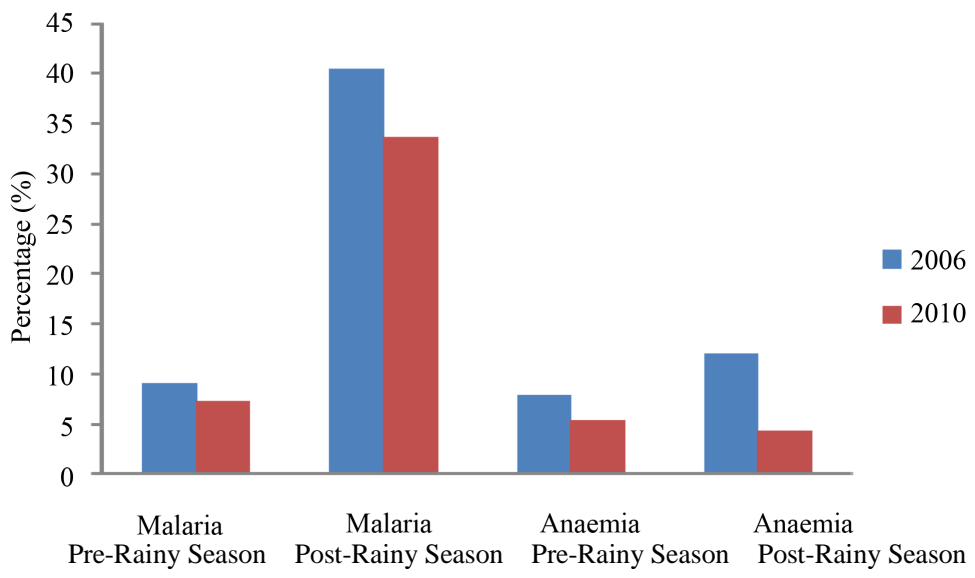

Figure 2. Comparison of malaria and anaemia prevalence between 2006 and 2010.

\section{Discussions}

The findings were derived from the analysis of the data obtained from the field. The findings were compared to similar studies done in different localities.

\subsection{Prevalence of Malaria}

Malaria burden in Hohoe municipality, as in most parts of Ghana, has remained high and in many areas even 
increased over the years. The main reason for this development is the rapid spread of resistance to commonly used monotherapies against malaria. This problem has necessitated urgent implementation of new and effective control strategies to "Roll Back Malaria." Two main interventions in this effort are the introduction of ACTs for treatment of uncomplicated malaria and the promotion of ITN use. The targets for the implementation of these new strategies have been defined by the UN Millennium Development Goals and the Abuja Declaration, to be achieved by the years 2015 and 2010, respectively. The ACTs were introduced in the Hohoe municipality in 2006 and ITNs were freely distributed during NIDs and are made available to the communities. The NMCP has regularly been supplying free ITNs to children under five years in the Hohoe municipality and Ghana as a whole from the Global fund.

The result of the study revealed that there has been a $20 \%$ reduction in the prevalence of malaria starting from 2006 to 2010 (from $9.0 \%$ to $7.2 \%$ ) at the beginning of the rainy season and a $16.8 \%$ reduction at the end of the rainy season (from 40.4\% to 33.6\%) (Figure 2). The two populations in 2006 and 2010 were similar with respect to their demographic characteristics and other social factors, hence the difference in the malaria prevalence may be associated with the malaria control interventions.

This goes to support the results of a study conducted in Zanzibar, Tanzania evaluating the impact of ACTs and ITNs and malaria trends after their implementation. Zanzibar implemented Artemisinin-based combination therapy (ACT) for uncomplicated malaria in late 2003 and LLINs from early 2006. Artemisinin-based combination therapy was provided free of charge to all malaria patients, while LLINs were distributed free to children under age 5 years and pregnant women. It was shown that $P$. falciparum prevalence decreased in children under five between 2003 and 2006 [15]. However, seasonal variations in the rainfall patterns over the years, environmental factors such as proper environmental management, presence of stagnant waters in the communities are contributory factors that can affect malaria prevalence. This study was done in June, hence comparable to the one done in June 2006. Behavioural change towards malaria awareness, the environmental changes by way of stagnant waters and water bodies could not be controlled for. The finding in this study also confirms the findings from a study that analyzed the impact of the introduction of LLINs and antimalarial medications among children in August 2006 in rural Rwanda [19].

The results showed that the absolute number of admissions due to suspected malaria was smaller during the post-intervention period relative to the pre-intervention period, in spite of an increase in the absolute number of hospitalizations due to other causes during the post-intervention period. The percentage of suspected malaria admissions that were laboratory-confirmed was greater during the pre-intervention period (80.4\%) relative to the post-intervention period (48.1\%).

A research conducted in United Kingdom showed that, when whole communities were provided with treated nets, the mosquito survival was reduced and so was the number of sporozoite-positive mosquitoes in malarious communities [20].

\subsection{Prevalence of Anaemia}

Malaria and anaemia are positively correlated, in that as malaria prevalence increases, anaemia prevalence also increases. Anaemia as a dependant variable has a number of independent variables affecting it. The independent variables include but not limited to the nutritional status of the children, the presence of helminthes, the existence of infectious disease, cancers and haemoglobinopathies. Significant reductions in anaemia prevalence have been demonstrated following malaria control programs in stable endemic areas, and usefulness of anaemia as an indicator of malaria burden and malaria control progress will depend on its specificity. The result of the study revealed that at the pre-rainy season survey prevalence of anaemia was reduced by 33\% from 2006 to 2010 [7.9\% vs. $5.3 \% ; \mathrm{p}<0.001$ ] and at the post-rainy season survey anaemia prevalence was significantly reduced by $64.2 \%$ [12\% vs. 4.3\%; $\mathrm{p}<0.001]$. This supports findings from a study that reported a $40 \%$ reduction of anaemia prevalence in 2004, and 21.7\% in 2005 (in the island of Biako in Equatorial Guinea after the introduction of ITNs and IRS) [21].

\subsection{ITN Ownership, Use and Effectiveness}

The role of ITNs in the control of malaria cannot be overemphasized. Owning an ITN does not protect you from malaria but rather its effective use and ability of the ITN to kill or prevent mosquitoes from biting individuals. Studies done in Ghana showed that, the use of ITNs are effective in reducing all cause malaria mortality and 
morbidity between $17 \%$ and $43 \%$ in children under five years and provides protection to them as they are the most susceptible to malaria infection [14]. This study shows that ownership of ITNs was about $65 \%$, use was $42 \%$ and effectiveness was 34.3\%. ITN ownership in the Hohoe municipality rose from $20.8 \%$ in 2006 to $64.8 \%$ in 2010 and ITN use rose from 15.1\% in 2006 to $42.2 \%$ in 2010 and effectiveness of ITNs rose from 20.0 in 2006 to 34.3\% in 2010 (Figure 1). Similarly a study in Ghana reported an increase in ITN ownership from 18\% in 2003 to $45 \%$ in 2008 and usage was $4.0 \%$ in 2003 to $28.0 \%$ in 2008 [11]. The ITNs being distributed in the municipality are not long lasting net and therefore require retreatment. In this study we found that only $34.3 \%$ of the nets were effective compared to $20.0 \%$ in 2006. This is not surprising because the nets being distributed by the malaria control programme are not Long Lasting Insecticide Nets (LLIN). However, there are lots of malaria community educations by the National Malaria Control Programme (NMCP) about the importance of ITNs, how to handle and retreat them and it is hoped that this would change their behavioural patterns and they will start using them in the correct ways.

\section{Conclusion}

Malaria morbidity is a major disease burden in children aged less than five in Ghana and Africa as a whole. It has both economic and social implications on the countries. In the Hohoe municipality, the malaria control interventions put in place in 2006 have shown a reduction in the prevalence of malaria by $20 \%$ and anaemia by $33 \%$ in 2010. It has also shown an increase in ITN ownership by $67.9 \%$, use by $64.2 \%$ and effectiveness by $41.7 \%$. Though the reduction was statistically significant, this has not yet met the national targets set in the Millennium Development Goals (MDGs) by the year 2015. The MDGs targets are to reduce the malaria burden by $75 \%$, and achieve a $100 \%$ ownership of ITN and $80 \%$ usage of ITN. However, in order to reach the MDG targets the promotion of ITNs usage and other preventive measures for malaria should be intensified.

\section{Recommendations}

Malaria control is a concerted effort that involves all stake holders. In line with this, the following measures are being recommended to the Hohoe Municipal Health Directorate (MHD):

Due the reduction in the prevalence of malaria in the municipality, the MHD should intensify its health education programmes on the importance of ITNs and use of ACTs for treating uncomplicated malaria. This will sustain the current prevalence levels or reduce it further.

ITN effectiveness (re-treatment) increased from 20.0\% to 34.3\% between 2006 and 2010. To achieve a higher effectiveness of ITNs and to avoid re-treatments, the MHD should promote the use of LLINs in the communities and support could be sought from the Municipal Assembly for LLINs promotion activities.

To achieve a greater reduction in malaria and anaemia prevalence than that obtained in 2010, new interventions like indoor residual spraying (IRS) can be given a second thought. The Municipal Director of Health Services can liaise with the NMCP for experts to come to evaluate the feasibility of this intervention. This method could have a long lasting effect on malaria control in the municipality.

Another cross sectional survey should be conducted at the beginning of the high transmission season in June and at the end of the high transmission season in November to see if there would be any significant association between malaria, anaemia and ITN use, ownership and effectiveness ten years after the introduction of the control interventions.

\section{Acknowledgements}

We are grateful to Dr. Micheal Ahedor, the Hohoe Municipal Health Management Team for their assistance during the survey. We are grateful to Mr King Kpo who monitored the quality of $\mathrm{Hb}$ measurement, slide preparation and reading. We are thankful to all the parents/guardian for giving.

\section{Conflict of Interest}

All authors report no conflict of interest in this study.

\section{Authors' Contributions}

MK worked on the 2006 project supported EA on the 2010 pre-rainy season study and continued with the 2010 
November survey. EA worked on the malaria programme at Hohoe as part of his project work for his MPH. All authors reviewed and approved the final version of the manuscript.

\section{References}

[1] Oresanya, O.B., Hoshen, M. and Sofola, O.T. (2008) Utilization of Insecticide-Treated Nets by Under-Five Children in Nigeria: Assessing Progress towards the Abuja Targets. Malaria Journal, 7, 145. http://dx.doi.org/10.1186/1475-2875-7-145

[2] Baume, C.A. and Marin, M.C. (2008) Gains in Awareness, Ownership and Use of Insecticide-Treated Nets in Nigeria, Senegal, Uganda and Zambia. Malaria Journal, 7, 153. http://dx.doi.org/10.1186/1475-2875-7-153

[3] (2007) Ghana Health Service/Ministry of Health Annual Report.

[4] (2008) Global Malaria Action Plan.

[5] (2007) World Malaria Report.

[6] (2008) World Health Organisation Report. Malaria Facts Sheet No. 94. http://www.who.int/mediacentre/factsheet

[7] (2014) Hohoe Municipal Health Directorate Annual Report.

[8] (2014) Hohoe Municipal Hospital Annual Report.

[9] Koram, K.A., Abuaku, B., Duah, N. and Quashie, N. (2005) Comparative Efficacy of Antimalarial Drugs including ACTs in the Treatment of Uncomplicated Malaria among Children under 5 Years in Ghana. Acta Tropica, 95, 194-203. http://dx.doi.org/10.1016/j.actatropica.2005.06.018

[10] (2005) Strategic Plan for Malaria Control in Ghana Handbook.

[11] (2008) Ghana Demographic and Health Survey.

[12] (2009) Ministry of Health Strategic Plan for Malaria Control in Ghana 2008-2015.

[13] (2009) Ministry of Health Anti-Malarial Drug Policy for Ghana.

[14] Binka, F. and Akweongo, P. (2006) Prevention of Malaria Using ITNs: Potential for Achieving the Millennium Development Goals. Current Molecular Medicine, 6, 261-267.

[15] Bhatttarai, A., Sali, A., Kachur, S.P., Martensson, A., Abbab, A.K., Khatid, R., Al-Mafazy, A.W., Ramsan, M., Rotllant, G., Gerstenmaier, J.F., Molteni, F., Abdulla, S., Montgomery, S., Kaneko, A. and Bjorkman, A. (2007) Impact of Artemisinin-Based Combination Therapy and Insecticide-Treated Nets on Malaria Burden in Zanziba. PLoS Medicine, 4, e309. http://dx.doi.org/10.1371/journal.pmed.0040309

[16] Delacollete, C., D’Souza, C., Chrstophel, E., Thimasaran, K., Abdur, R., Bell, D., Dai, T.C., Gopinath, D., Lu, S., Mendoza, R., Ortega, L., Rastrogi, R., Tantinimitkul, C. and Ehrenberg, J. (2009) Malaria Trends and Challenges in the Greater Mekoy Subregion. Southeast African Journal Tropical Medicine Public Health, 40, 674-691.

[17] Kweku, M., Liu, D., Adjuik, M., Binka, F., Seidu, M., Greenwood, B. and Chandramohan, D. (2008) Seasonal Intermittent Preventive Treatment for the Prevention of Anaemia and Malaria in Ghanaian Children: A Randomized, Placebo Controlled Trial. PLoS ONE, 3, e4000. http://dx.doi.org/10.1371/journal.pone.0004000

[18] (2010) Ghana National Population Census.

[19] Sievers, A.C., Lewey, J., Musafir, I.P., Fianke, M.F., Bucyibaruta, B.J., Stulac, S.N., Rich, M.L., Karema, C. and Daily, J.P. (2008) Reduced Paediadtic Hospitalizations for Malaria and Febrile Illness Patterns Following Implementation of Community-Based Malaria Control Programme in Rural Rwanda. Malaria Journal, 7, 167. http://dx.doi.org/10.1186/1475-2875-7-167

[20] Curtis, C.F., Maxwell, C.A., Magesa, S.M., Rwegoshora, R.T. and Wikes, T.J. (2006) Insecticide-Treated Bed-Nets for Malaria Mosquito Control. Journal of the American Mosquito Control Association, 22, 501-506. http://dx.doi.org/10.2987/8756-971X(2006)22[501:IBFMMC]2.0.CO;2

[21] Pardo, G., Descalzo, M.A., Molina, L., Custodio, E., Lwanga, M., Mangue, C., Obono, J., Nchama, A., Roche, J., Benito, A. and Cano, J. (2006) Impact of Different Strategies to Control Plasmodium Infection and Anaemia on the Island of Bioko (Equitorial Guinea). Malaria Journal, 5, 10. http://dx.doi.org/10.1186/1475-2875-5-10 